\title{
Correction to: A suggestion to introduce the diagnosis of "diffuse midline glioma of the pons, H3 K27 wildtype (WHO grade IV)"
}

\author{
André O. von Bueren ${ }^{1,2,3}$ - Michael Karremann ${ }^{4} \cdot$ Gerrit H. Gielen $^{5} \cdot$ Martin Benesch $^{6} \cdot$ Maryam Fouladi $^{7}$. \\ Dannis G. van Vuurden ${ }^{8,9}$. Sophie E. M. Veldhuijzen van Zanten ${ }^{8,9} \cdot$ Lindsey M. Hoffman $^{10,11}$. Christof M. Kramm ${ }^{3}$
}

Published online: 16 August 2018

(c) Springer-Verlag GmbH Germany, part of Springer Nature 2018

\section{Correction to: Acta Neuropathologica (2018) 136:171-173 https://doi.org/10.1007/s00401-018-1863-6}

The citation of the original publication in PubMed contains an error. The seventh author name is wrongly cited.

It should be cited as "von Bueren AO, Karremann M, Gielen GH, Benesch M, Fouladi M, van Vuurden DG, Veldhuijzen van Zanten SEM, Hoffman LM, Kramm CM".

The original article can be found online at https://doi.org/10.1007/ s00401-018-1863-6.

André O. von Bueren

andre.vonburen@hcuge.ch

$\triangle$ Christof M. Kramm

christof.kramm@med.uni-goettingen.de

1 Department of Pediatrics and Adolescent Medicine, Division of Pediatric Hematology and Oncology, University Hospital of Geneva, Geneva, Switzerland

2 Department of Pediatrics, CANSEARCH Research Laboratory, Faculty of Medicine, University of Geneva, Geneva, Switzerland

3 Division of Pediatric Hematology and Oncology, University Medical Center Goettingen, Goettingen, Germany

4 Department of Pediatric and Adolescent Medicine, University Medical Center Mannheim, Medical Faculty Mannheim, Heidelberg University, 68167 Mannheim, Germany
5 Institute of Neuropathology, University Hospital Bonn, Bonn, Germany

6 Department of Pediatrics and Adolescent Medicine, Division of Pediatric Hematology and Oncology, University of Graz, Graz, Austria

7 Cincinnati Children's Hospital Medical Center, Cancer and Blood Diseases Institute, Cincinnati, OH, USA

8 Department of Neuro-Oncology, Princess Máxima Center for Pediatric Oncology, Utrecht, The Netherlands

9 Department of Pediatrics, Division of Oncology, VU University Medical Center, Amsterdam, The Netherlands

10 Department of Pediatrics, University of Colorado Denver, Anschutz Medical Campus, Aurora, CO, USA

11 Morgan Adams Foundation Pediatric Brain Tumor Research Program, Children's Hospital Colorado, Aurora, CO, USA 